Pacific Journal of Mathematics

THE ORIENTABILITY OF MATCHBOX MANIFOLDS

Jan darTS, CHARLES Lemuel Hagopian 


\title{
THE ORIENTABILITY OF MATCHBOX MANIFOLDS ${ }^{1}$
}

\author{
J. M. Aarts, C. L. Hagopian, and L. G. Oversteegen
}

A separable and metrizable space $X$ is a matchbox manifold if each point $x$ of $X$ has an open neighborhood which is homeomorphic to $S_{x} \times \mathbb{R}$ for some zero-dimensional space $S_{x}$. Each arc component of a matchbox manifold admits a parameterization by the reals $\mathbb{R}$ in a natural way. This is the main tool in defining the orientability of matchbox manifolds. The orientable matchbox manifolds are precisely the phase spaces of one-dimensional flows without rest points. We show in this paper that a compact homogeneous matchbox manifold is orientable.

As an application a new proof is given of Hagopian's theorem that a homogeneous metrizable continuum whose only proper nondegenerate subcontinua are arcs must be a solenoid. This is achieved by combining our work on matchbox manifolds with Whitney's theory of regular curves.

1. Introduction. In our discussion we need some definitions and results from the papers [1] and [2] in which flows without rest points on one-dimensional spaces were discussed. A separable metric space $X$ is called a matchbox manifold if for each point $x$ of $X$ there is a zero-dimensional space $S_{x}$ such that $S_{x} \times \mathbb{R}$ is homeomorphic to an open neighborhood of $x$.

For any zero-dimensional subspace $S$ of $\mathbb{R}$ we let

$$
\begin{aligned}
& F_{S}=\left\{(x, y) \in \mathbb{R}^{2} \mid x \in S,-1 \leq y \leq 1\right\} \quad \text { and } \\
& E_{S}=\left\{(x, y) \in \mathbb{R}^{2} \mid x \in S,-1<y<1\right\} .
\end{aligned}
$$

If $h: F_{S} \rightarrow X$ is a topological embedding such that $h\left(F_{S}\right)$ is closed and $h\left(E_{S}\right)$ is open in $X$, then $V=h\left(F_{S}\right)$ is called a matchbox in $X$. In this case we also say that $V$ is a matchbox neighborhood of $h(x, 0), x \in S$. The induced map $h: F_{S} \rightarrow V$ is called a parameterization of $V$. In a matchbox manifold every point has arbitrarily small matchbox neighborhoods. As orientability of matchbox manifolds is the main topic of our paper, we briefly discuss its definition. First parameterizations of arc components are defined. If an arc component $C$ is compact, it is a circle and any covering map of $\mathbb{R}$ to $C$

${ }^{1}$ These results were announced at the sixth Brasilean Topology Conference, Campinas, Brasil, August 15-19, 1988. 
is called a parameterization. If the arc component $C$ is non-compact, then any continuous bijection $p: \mathbb{R} \rightarrow C$ is called a parameterization. The most important property of parameterizations is the so-called arc lifting property formulated in the following lemma. See [1], [3] for details.

LemMA. Suppose that $p_{1}$ and $p_{2}$ are parameterizations of an arc component of a match manifold $X$. Then for each $a_{1}$ and $a_{2}$ such that $p_{1}\left(a_{1}\right)=p_{2}\left(a_{2}\right)$ there is a unique homeomorphism $h: \mathbb{R} \rightarrow \mathbb{R}$ such that $p_{2}=p_{1} \circ h$ and $h\left(a_{2}\right)=a_{1}$.

Because any homeomorphism of $\mathbb{R}$ onto itself is either decreasing or increasing, it is clear that the parameterizations of an arc component fall into two classes, the directions. Now if $X$ is a matchbox manifold, we let $\left\{C_{\alpha} \mid \alpha \in A\right\}$ denote the collection of arc components. If $p_{\alpha}: \mathbb{R} \rightarrow C_{\alpha}$ is a parameterization for each $\alpha \in A$, then the collection $\left\{p_{\alpha} \mid \alpha \in A\right\}$ is called a parameterization of $X$. Now let $V$ be a matchbox in $X$ with parameterization $h: F_{S} \rightarrow V$. As before, $F_{S}=S \times[-1,1]$ for some zero-dimensional subset $S$ of $\mathbb{R}$. By pr we denote the projection of $F_{S}$ onto $[-1,1]$. As in [2] we shall say that $V$ is coherently directed by the parameterization $\left\{p_{\alpha} \mid \alpha \in A\right\}$ of $X$ if for each $x \in S$ and for any closed interval $J$ in $\mathbb{R}$ with $p_{\alpha}(J)=h(\{x\} \times[-1,1])$ for some $\alpha$ the composition pr $\circ h^{-1} \circ p_{\alpha}$ is increasing.

Finally, the matchbox manifold $X$ is said to be orientable if there exists a parameterization $\left\{p_{\alpha} \mid \alpha \in A\right\}$ of $X$ such that each point has a matchbox neighborhood which is coherently directed.

By the characterization theorem of [2] a separable metrizable space $X$ is an orientable matchbox manifold if and only if $X$ is the phase space of some one-dimensional flow without rest points. In [1] and [2] examples have been presented of matchbox manifolds which are not orientable. See $\S 5$ for more examples. It is the main result of this paper that if the matchbox manifold is a homogeneous compact space, then it must be orientable. Recall that a space $X$ is said to be homogeneous if for all $x$ and $y$ in $X$ there is a homeomorphism $h: X \rightarrow X$ such that $h(x)=y$.

MAIN TheOREM. If a matchbox manifold is compact and homogeneous, then it is orientable.

In $\S 5$ by means of Examples 2 and 3 we shall show that both the ingredients compact and homogeneous are needed in the theorem. 
The proof of the main theorem and its corollary are presented in $\S 2$. In $\S 3$ we discuss a modification of an important result of Whitney [17] about the local product structure of regular families of curves. This is a preparation for the application of the main theorem to the situation of a homogeneous continuum all proper subcontinua of which are arcs. As a consequence we obtain a new proof of Hagopian's theorem [11] that such a continuum must be a solenoid. Another proof of this result was recently obtained by Mislove and Rogers [12]. See $\S 4$.

2. Proof of main result. We shall frequently use Effros' theorem [9] (see also [5]). We say that a metric space $X$ (with metric $d$ ) has the Effros property if for every $\varepsilon>0$ there exists a $\delta>0$ such that for all points $y$ and $z$ in $X$ satisfying $d(y, z)<\delta$ there is a homeomorphism $h: X \rightarrow X$ such that $h(y)=z$ and $d(x, h(x))<\varepsilon$ for all $x \in X$. In this situation we say that $\delta$ is an Effros delta for $\varepsilon$. As a consequence of Effros' theorem each compact homogeneous space has the Effros property.

Proof of the main theorem. Let $X$ be a matchbox manifold which is compact and homogeneous. In [4] it has been shown that every homogeneous locally compact separable metrizable space is the product of a connected space and a zero-dimensional space. So without loss of generality we may assume that $X$ is connected. Let $\left\{C_{\alpha} \mid \alpha \in A\right\}$ denote the collection of all arc components of $X$. Let $x$ be a point in $X$ and let $V$ be any matchbox neighborhood of $x$ with parameterization $h: F_{S} \rightarrow V$.

First we shall show that there is a parameterization $\left\{p_{\alpha} \mid \alpha \in A\right\}$ of $X$ and a subset $S_{1}$ of $S$ such that $V_{1}=h\left(F_{S_{1}}\right)$ is a matchbox neighborhood of $x$ which is coherently directed by $\left\{p_{\alpha} \mid \alpha \in A\right\}$. We assume that this is not true and we shall derive a contradiction. From the assumption it follows that there exists a sequence $\left(\delta_{n}\right)$ of positive numbers, which converges to 0 , and that there exist for each $n \in \mathbb{N}$ points $y_{n}, z_{n} \in S$ and intervals $I_{n}$ and $J_{n}$ in $\mathbb{R}$ such that

(1) $h\left(\left\{y_{n}\right\} \times[-1,1]\right)=p_{\alpha_{n}}\left(I_{n}\right)$,

(2) $h\left(\left\{z_{n}\right\} \times[-1,1]\right)=p_{\alpha_{n}}\left(J_{n}\right)$,

(3) the map proh-1 $\circ p_{\alpha_{n}}$ is increasing on one of the intervals $I_{n}$ and $J_{n}$, and decreasing on the other of $I_{n}$ and $J_{n}$,

(4) $d\left(h\left(y_{n}, 0\right), x\right)<\frac{1}{2} \delta_{n}, d\left(h\left(z_{n}, 0\right), x\right)<\frac{1}{2} \delta_{n}$.

We shall write $C_{\alpha_{n}}=p_{\alpha_{n}}(\mathbb{R})$. By taking a suitable subsequence we may also assume that

(5) $\delta_{n}$ is an Effros delta for $\frac{1}{n+1}$. 
Let $\varepsilon$ be the minimum of the numbers

$$
\begin{aligned}
& d(h(S \times\{1 / 2\}), X-h(S \times(0,1))) \text { and } \\
& d(h(S \times\{-1 / 2\}), X-h(S \times(-1,0))) .
\end{aligned}
$$

As $X$ is compact, $\varepsilon$ is positive. For every $n \in \mathbb{N}$ we find a homeomorphism $g_{n}: X \rightarrow X$ such that

$$
\begin{aligned}
& g_{n}\left(h\left(y_{n}, 0\right)\right)=h\left(z_{n}, 0\right) \text { and } \\
& d\left(g_{n}(u), u\right)<\frac{1}{n+1} \quad \text { for all } u \in X .
\end{aligned}
$$

It is clear that $g_{n}$ maps $C_{\alpha_{n}}$ onto itself. Without loss of generality we may assume that pro $h^{-1} \circ p_{\alpha_{n}}$ is increasing on $I_{n}$ and decreasing on $J_{n}$.

From the definition of $\varepsilon$ it follows that for any $n$ with $1 /(n+1)<\varepsilon$ the mapping

$$
t \rightarrow \operatorname{pr} \circ h^{-1} \circ g_{n} \circ h\left(y_{n}, t\right)
$$

is increasing. For any such $n$ the map proh-1 $\circ g_{n} \circ p_{\alpha_{n}}$ is defined on some subinterval $I$ of $I_{n}$ and this map is increasing on $I$.

The map $g_{n} \circ p_{\alpha_{n}}$ is a parameterization of $C_{\alpha_{n}}$ and by the lemma in $\S 1$ there is a homeomorphism $f_{n}: \mathbb{R} \rightarrow \mathbb{R}$ such that

$$
g_{n} \circ p_{\alpha_{n}}=p_{\alpha_{n}} \circ f_{n} .
$$

Note that $f_{n}$ sends $I$ into $J_{n}$. It follows that the map

$$
\operatorname{pr} \circ h^{-1} \circ g_{n} \circ p_{\alpha_{n}}=\operatorname{pr} \circ h^{-1} \circ p_{\alpha_{n}} \circ f_{n}
$$

is increasing. As pro $h^{-1} \circ p_{\alpha_{n}}$ is decreasing on $J_{n}$, we see that $f_{n}$ is decreasing on $I$. It has been proved now that $f_{n}$ is decreasing on $\mathbb{R}$. We denote the unique fixed point of $f_{n}$ by $t_{n}$ and we write $p_{n}=$ $p_{\alpha_{n}}\left(t_{n}\right)$. We see that $g_{n}\left(p_{n}\right)=p_{n}$. In this way we find a point $p_{n}$ for each $n$ satisfying $1 /(n+1)<\varepsilon$. By compactness of $X$ we may assume that the sequence $\left(p_{n}\right)$ converges to $p$. Let $V^{*}$ denote a matchbox neighbor of $p$ with parameterization $h^{*}: F_{S^{*}} \rightarrow V^{*}$. Define $\varepsilon^{*}$ in a similar way as $\varepsilon$ above. Because $f_{n}$ is decreasing, the maps $g_{n}$ move some points of $V^{*}$ more than $\frac{1}{2} \varepsilon^{*}$, for all $n$ with $1 /(n+1)<$ $\varepsilon^{*}$. Since $g_{n} \rightarrow$ id, the maps $g_{n}$ move no point more than $\frac{1}{2} \varepsilon^{*}$ for sufficiently large $n$. This is a contradiction.

Having established that there exists a matchbox $V_{1}$ and a parameterization $\left\{p_{\alpha} \mid \alpha \in A\right\}$ of $X$ such that $V_{1}$ is coherently directed by $\left\{p_{\alpha} \mid \alpha \in A\right\}$, we now show that $X$ is orientable. As in [2] we denote 
by $B\left(V_{1}\right)$ the union of all arc components of $X$ which have a nonempty intersection with $V_{1}$. By using the lemma of the long box of [2] we see that $B\left(V_{1}\right)$ is an open subset of $X$ which is orientable by the very same parameterization. The proof is completed by showing that $X=B\left(V_{1}\right)$. As $X$ is assumed to be connected, we need only show that $B\left(V_{1}\right)$ is closed. Let $q \in \operatorname{cl} B\left(V_{1}\right)$. For every natural number $n$ we select $q_{n} \in B\left(V_{1}\right)$ and $x_{n} \in S_{1}$ such that $q_{n} \rightarrow q$ and $h\left(x_{n}, 0\right)$ and $q_{n}$ are in the same arc component. As $V_{1}$ is compact, we may assume that $x_{n} \rightarrow x^{*} \in S_{1}$. As $h\left(x^{*}, 0\right) \in h\left(E_{S_{1}}\right)$ and $h\left(E_{S_{1}}\right)$ is open, there is an $\eta<0$ such that

$$
B_{2 \eta}\left(h\left(x^{*}, 0\right)\right) \subset h\left(E_{S_{1}}\right) \text {. }
$$

For this $\eta$ we choose an Effros delta $\delta$. For $n$ with $d\left(q_{n}, q\right)<\delta$ and $d\left(h\left(x_{n}, 0\right), h\left(x^{*}, 0\right)\right)<\delta$ we find that the distance of $h\left(x_{n}, 0\right)$ to the arc component of $q$ is less than $\eta$. It follows that the arc component of $q$ hits $h\left(E_{S_{1}}\right)$ and thus $q \in B\left(V_{1}\right)$.

Since $V_{1}$ is an arbitrarily small matchbox neighborhood in the last part of the proof, namely the proof of $X=B\left(V_{1}\right)$, we have established the following corollary.

COROLLARY. If a matchbox manifold is compact, homogeneous and connected, then each of its arc components is dense.

3. Local product structure. In this section $X$ is a separable metrizable space such that every proper nondegenerate subcontinuum of $X$ is an arc. For each $x \in X$ we let $C_{x}$ denote the arc component of $X$. By our assumption of $X$ the set $C_{X}$ is the union of all proper subcontinua of $X$ containing $x$. Obviously, for all $x, y \in X$ either $C_{x}=C_{y}$ or $C_{x} \cap C_{y}=\varnothing$. For every $x \in X$ the set $C_{x}$ is a one-to-one continuous image of either $[0,1],(0,1)$ or $[0,1)$ and, consequently, inherits an order. We want to emphasize here that $C_{x}$ may fail to be a topological image of an interval. We shall say that the family $\left\{C_{x} \mid x \in X\right\}$ of arc components is regular if for each $x \in X$, for each arc $I \subset C_{x}$, containing $x$, and for each $\varepsilon>0$ there exists a $\delta>0$ such that for each $y \in B_{\delta}(x)$ there exists an $\varepsilon$-embedding $h: I \rightarrow C_{y}$ (i.e., $d(u, h(u))<\varepsilon$ for each $u \in I$ ) such that $h(x)=y$. For $a, b \in C_{x}$ we shall denote the arc joining $a$ and $b$ by $[a, b]$. We write $(a, b)=[a, b] \backslash\{a, b\}$.

In $\S 5$, Example 3, we present an example of a space $X$ with a nontrivial regular family of arc components which consists of just one element. 
The main goal of this section is to prove the existence of local sections and to exhibit the local product structure when the arc components form a regular family. Results of this type are often obtained under more restrictive hypotheses. For example, Bebutov [15, p. 333], makes use of the group property in dynamical systems. We shall follow an argument designed by Whitney [17] for a different situation. The Whitney function $\mu$ will play the role of a potential function.

By $2^{X}$ we denote the space of closed subsets of $X$ endowed with the Hausdorff metric [14]. It is known that $2^{X}$ is a separable metric space. Let $\mu: 2^{X} \rightarrow \mathbb{R}^{+} \cup\{0\}$ be a Whitney function of $2^{X},[17, \mathbf{p}$. 246]. That is, $\mu$ is a continuous function such that $\mu(\{x\})=0$ for each $x \in X$ and, if $A$ is a proper closed subset of the closed set $B$, then $\mu(A)<\mu(B)$.

Now suppose that the family of arc components of $X$ is regular. Let $x \in X$. We say that the closed set $S$ is a local section at $x$ provided that $x \in S$ and that there exists a neighborhood $U$ of $x$ such that for each $y \in \bar{U}$ each component of $C_{y} \cap \bar{U}$ intersects $S$ in exactly one point.

LEMMA. Let $X$ be a separable metrizable space such that the family of arc components of $X$ is regular. Then $X$ admits a local section at every point $x \in X$ such that $x$ is contained in some arc $(a, b)$ in $C_{x}$.

Proof. Without loss of generality we may assume that there are $c, d$ in $C_{x}$ such that $x \in(a, b) \subset[a, b] \subset(c, d) \subset[c, d] \subset C_{x}$. Let $<$ denote an order on $[c, d]$ such that $c<d$. As the family of arc components is regular, there exist $0<\delta<\eta$ and a neighborhood $V$ of $a$ such that

(i) $\bar{V} \subset B_{\eta}(a)$;

(ii) $B_{2 \eta}(a) \cap B_{2 \delta}(x)=\varnothing$;

(iii) for each $y \in B_{2 \delta}(x)$ there exists an embedding $h:[c, d] \rightarrow C_{y}$ such that $h(x)=y$ and $h(a) \in V$; this embedding $h$ induces an order $<$ on $h([c, d])$ such that $h(c)<h(d)$ and if $z_{1}, z_{2} \in \bar{V} \cap h([c, d])$, then $\left[z_{1}, z_{2}\right] \subset B_{\eta}(a)$.

For each $y \in \bar{B}_{\delta}(x)$ we let

$$
a_{y}=\sup \left\{z \in C_{y} \mid z \in \bar{V} \text { and } z<y\right\}
$$

and

$$
T_{y}=\left[a_{y}, y\right] \cup \bar{B}_{\eta}(a) .
$$


The map $y \rightarrow T_{y}$ is a continuous map $\bar{B}_{\delta}(x) \rightarrow 2^{X}$. Consequently the map $\varphi: \bar{B}_{\delta}(x) \rightarrow \mathbb{R}^{+}$, defined by $\varphi(y)=\mu\left(T_{y}\right)$, is a continuous function which is strictly increasing on arc components of $C_{y} \cap \bar{B}_{\delta}(x)$, $y \in \bar{B}_{\delta}(x)$. Choose $x_{i}$ and a neighborhood $V_{i}$ of $x_{i}, i=1,2$ such that

(i) $a<x_{1}<x<x_{2}<b$,

(ii) $x \notin \bar{V}_{1} \cup \bar{V}_{2}$,

(iii) $\bar{V}_{1} \cup\left[x_{1}, x_{2}\right] \cup \bar{V}_{2} \subset B_{\delta}(x)$, and

(iv) for each $y_{i} \in \bar{V}_{i}, i=1,2, \varphi\left(y_{i}\right)<\varphi(x)<\varphi\left(y_{2}\right)$.

Now let $U=\left\{y \in B_{\delta}(x) \mid\right.$ there exist $y_{i} \in V_{i}, i=1,2$ such that $y_{1}<$ $y<y_{2}$ and $\left.\left[y_{1}, y_{2}\right] \subset B_{\delta}(x)\right\}$. Since the family of arc components of $X$ is regular, $U$ is a neighborhood of $x$. Define

$$
S=\{w \in \bar{U} \mid \varphi(w)=\varphi(x)\} .
$$

Then for each $y \in \bar{U}$ each component of $C_{y} \cap \bar{U}$ intersects $S$ in exactly one point.

THEOREM. Let $X$ be a separable metrizable space such that the family of arc components of $X$ is regular. Suppose that $x$ is contained in some arc $(a, b) \subset C_{x}$. then there exist a neighborhood $U$ of $x, a$ section $S$ at $x$ and an embedding $\psi: S \times[-1,1] \rightarrow \bar{U}$ such that

(i) $\psi(s, 0)=s$ for every $s \in s$.

Proof. We may assume that $[a, b] \subset C_{x}$. Let $<$ be an order on $[a, b]$ such that $a<b$. By the preceding lemma $X$ has a local section $S^{\prime}$ at $x$. Hence there exists a neighborhood $V$ of $x$ such that for each $y \in \bar{V}$ there exists a unique $s_{y} \in S^{\prime} \cap C_{y}$ such that $\left[y, s_{y}\right]$ or $\left[s_{y}, y\right]$ is contained in $\bar{V}$. Choose $\delta<0$ such that $B_{2 \delta}(a) \cap$ $\left[[x, b] \cup B_{\delta}(b)\right]=\varnothing$ and $B_{2 \delta}(b) \cap\left[[a, x] \cup B_{\delta}(a)\right]=\varnothing$. Choose $\eta>0$ such that $\bar{B}_{\eta}(x) \subset V$ and for each $y \in \bar{B}_{\eta}(x)$ there exists a $\delta$-embedding $h:[a, b] \rightarrow C_{y}$ such that

$$
\begin{gathered}
h(x)=y, \quad \mu\left(\left[h(a), s_{y}\right]\right)>\frac{\mu([a, x])}{2}, \quad \text { and } \\
\mu\left(\left[s_{y}, h(b)\right]\right)>\frac{\mu([x, b])}{2} .
\end{gathered}
$$

The map $h$ induces an order on $h([a, b])$ such that $h(a)<h(b)$.

Now let $S=\left\{s_{y} \mid y \in B_{\eta}(x)\right\}, I=[-1,1]$ and define

$$
\psi: S \times I \rightarrow \psi(S \times I)=\bar{U}
$$


by

$$
\psi(s, t)=y \quad \text { where } \begin{cases}y \leq s \text { satisfies } \frac{2 \mu([y, s])}{\mu([a, x])}=|t|, & \text { if } t \leq 0, \\ y \geq s \text { satisfies } \frac{2 \mu([s, y])}{\mu([x, b])}=t, & \text { if } t \geq 0 .\end{cases}
$$

Obviously $\psi$ is one-to-one and onto $\psi(S \times I)$. The continuity of $\psi$ follows from the continuity of $\mu$. Suppose that $y_{i} \rightarrow y_{0}$. Then $s_{y_{2}} \rightarrow s_{y_{0}}$ and $\lim \left[s_{y_{2}}, y_{i}\right]=\left[s_{y_{0}}, y_{0}\right]$. Hence $\mu\left(\left[s_{y_{i}}, y_{i}\right]\right)=t_{i} \rightarrow$ $\mu\left(\left[s_{y_{0}}, y_{0}\right]\right)=t_{0}$ and $\left(s_{y_{\imath}}, t_{i}\right) \rightarrow\left(s_{y_{0}}, t_{0}\right)$.

COROllary. Let $X$ be a one-dimensional separable metrizable space such that the family of arc components of $X$ is regular. Suppose that $x$ is contained in some arc $(a, b) \subset C_{x}$. Then there is $a$ matchbox neighborhood of $x$ in $X$.

Proof. By the theorem there exists a section $S$ and an embedding $\psi: S \times I \rightarrow \bar{U}$ where $U$ is a neighborhood of $x$. As $\operatorname{dim} S \times I \leq 1$, we must have $\operatorname{dim} S=0$ [10,1.9.E]. Let $S^{\prime}$ be a clopen subset of $S$ containing $x$ such that $\psi\left(S^{\prime} \times I\right) \subset$ int $U$. Then $h\left(S^{\prime} \times I\right)$ is a matchbox in $X$.

COROLlaRY. Let $X$ be a compact metrizable space such that the family of arc components of $X$ is regular. Suppose that $x$ is contained in some arc $(a, b) \subset C_{x}$. Then there is a matchbox neighborhood of $x$ in $X$.

Proof. By the theorem there is a section $S$ and an embedding $\psi: S \times I \rightarrow \bar{U}$ where $U$ is a neighborhood of $x$. Now $S$ must be totally disconnected, because otherwise $X$ would contain a continuum of dimension greater than one. That however is impossible. As $S$ is compact, it follows that $\operatorname{dim} S=0$.

4. Applications. In this section we shall show that every homogeneous continuum such that every proper nondegenerate subcontinuum is an arc admits a flow without rest points. This answers a question of Hagopian. Using a result of Thomas [16] we obtain an alternative proof of Hagopian's theorem that such a continuum is a solenoid [11], [12]. We will always assume that $X$ is a nondegenerate continuum.

LEMMA 4.1. Let $X$ be a homogeneous compact metrizable space such that every proper subcontinuum is an arc. Then $X$ is an orientable matchbox manifold. 
Proof. It follows from Effros' theorem that the family of arc components is regular. Also because of homogeneity each point $x$ of $X$ is contained in the interior of an arc in the arc component $C_{x}$. By the second corollary in $\S 3$ the space $X$ is a matchbox manifold. By the main theorem $X$ is an orientable manifold.

THEOREM. Let $X$ be a homogeneous continuum such that every proper subcontinuum is an arc. Then $X$ admits a flow $\varphi: X \times \mathbb{R} \rightarrow X$. Moreover the arc component of each point $x \in X$ coincides with the orbit of $x$ and hence $X$ is minimal under $\varphi$.

Proof. By the lemma $X$ is an orientable matchbox manifold. Hence by [2] $X$ admits a flow such that the arc components coincide with the orbits. By the corollary in $\S 2$ we get that the orbits are dense. Hence, $X$ is minimal under $\varphi$.

CoRollaRY (Hagopian [11]). Let $X$ be a homogeneous continuum such that every proper subcontinuum is an arc; then $X$ is a solenoid.

Proof. By the theorem $X$ admits a minimal flow $\varphi$ such that for each point $x$ the arc component of $x$ and the orbit of $x$ coincide. Let $x \in X$ and let $V=h\left(F_{S}\right)$ be a matchbox neighborhood of $x$. We write $Z=h(S \times\{0\})$, the zero level of $V$. The first return map $r: Z \rightarrow Z$ is defined by $r(x)=\varphi\left(x, t_{0}\right)$, where $t_{0}=$ $\inf \{t>0 \mid \varphi(x, t) \in Z\}$. It is to be observed that $r$ is well-defined because $\varphi$ is minimal and $h\left(E_{S}\right)$ is open [16, p. 234]. In [16] Thomas has shown that certain flows are solenoids. The exact same proof of Thomas' paper can be used after the following modification has been made. In the key lemma for Theorem 1 [16, in paragraph 5 on page 126], it is observed that the family $\left\{r^{n} \mid n \geq 0\right\}$ of iterates of $r$ is equicontinuous.

The proof of this fact should be replaced by the following argument.

The map $\pi: h\left(F_{S}\right) \rightarrow Z$ is defined by $\pi(h(x, t))=h(x, 0), x \in S$. Let $\gamma$ be the minimum of $d\left(h\left(Z \times\left\{\frac{1}{2}\right\}\right), X-h(Z \times(0,1))\right)$ and $d\left(h\left(Z \times\left\{-\frac{1}{2}\right\}\right), X-h(Z \times(-1,0))\right)$. Let $\varepsilon>0$ be given. First choose $\eta<\gamma$ such that for each $p \in Z$ and each $q \in B_{\eta}(p)$ we have $d(p, \pi(q))<\varepsilon$. Let $\delta$ be an Effros delta for $\eta$. We claim that

$$
d\left(r^{n}(x), r^{n}(y)\right)<\varepsilon \text { for each } n \geq 0 \text { and all } x \text { and } y \text { in } Z
$$

with $d(x, y)<\delta$.

Let $x, y \in Z$ such that $d(x, y)<\delta$. Let $g: X \rightarrow X$ be a homeomorphism such that $g(x)=y$ and $d(y, g(u))<\eta$ for all $u \in X$. 
By the choice of $\gamma$ it follows that for all sufficiently small $t>0$, $g(h(x, t))=h(y, s)$, for some $s>0$. Using the order by time on orbits, we see that $g$ is increasing.

Let $n>0$. We have

$$
g\left(r^{n}(x)\right) \in B_{\eta}\left(r^{n}(x)\right) \subset h\left(E_{S}\right) .
$$

It follows that

$\pi \circ g \circ r^{n}(x)=r^{m}(y)$ for some $m \geq 0$ and $d\left(r^{n}(x), r^{m}(y)\right)<\varepsilon$.

In a similar way it follows that for some $\eta$-homeomorphism $g^{\prime}$ we have for all $n>0$, $\pi \circ g^{\prime} \circ r^{n}(y)=r^{m}(x)$ for some $m \geq 0$ and $d\left(r^{n}(y), r^{m}(x)\right)<\varepsilon$. An easy inductive argument shows that always $m=n$. Consequently $\left\{r^{n} \mid n \geq 0\right\}$ is equicontinuous.

This completes the proof of the corollary.

5. Examples. Now we present some examples which may clarify the discussion.

EXAMPLE 1. The pseudo-arc $[7,13]$ and the universal curve [6] are examples of compact homogeneous spaces without a matchbox structure. The pseudo-arc does not admit any flow because it contains no arcs. Because the universal curve has a dense collection of arbitrarily small closed curves, it only admits the trivial flow in which each point is a rest point.

EXAMPLE 2. We present a compact matchbox manifold $X$ which is not orientable. Using polar coordinates we let

$$
\begin{aligned}
Y=\{ & (1, \varphi) \mid 0 \leq \varphi<2 \pi\} \cup\{(2, \varphi) \mid 0<\varphi \leq 2 \pi\} \\
& \cup\{(r, \varphi) \mid \varphi \in \mathbb{R}, r=\psi(\varphi)\},
\end{aligned}
$$

which $\psi$ is a strictly increasing continuous function of $\mathbb{R}$ onto $(1,2)$. $X$ is obtained by identifying the points

$$
(1, \varphi) \text { and }(2,2 \pi-\varphi), \quad 0 \leq \varphi<2 \pi .
$$

ExAmple 3. Let $E$ denote the set of endpoints of the Cantor set $C$; i.e., $E$ is the set of points in $[0,1]$ the triadic expansion of which has no 1's and eventually either 0 's or 2's. The space $X^{*}$ consists of all semicircles in the upper half of the plane with center $\left(\frac{1}{2}, 0\right)$ through the points of $E$ and of all semicircles in the lower half of 
the plane with center $\left(5 / 2.3^{n}, 0\right)$ through the points $x$ of $E$ such that $2 / 3^{n} \leq x \leq 1 / 3^{n-1}, n \geq 1 . \quad X^{*}$ is just an arc component of the well-known Knaster bucket handle. $\left\{X^{*}\right\}$ is a regular family of arc components. The space $X=X^{*} \backslash\{(0,0)\}$ is a homogeneous matchbox manifold which is not orientable [1], p. 48.

Related to Example 3 we have the following question.

Question. Does there exist a homogeneous curve (i.e. one-to-one continuous image of the real line) that is not a matchbox manifold?

EXAMPLe 4. On the two dimensional torus we consider the Denjoy modification of the irrational flow [15, p. 381]. Let $X$ be the minimal set in this flow. Since $X$ is embedded in a 2-manifold, $X$ is not homeomorphic with a solenoid [8]. From the corollary of $\S 4$ we may conclude that $X$ is not homogeneous.

EXAMPLE 5. Let $Y$ be an arc component of the space $X$ in Example 4. As $Y$ is an orbit in a flow, $Y$ is homogeneous. $Y$ is a matchbox manifold, but fails to be compact.

\section{REFERENCES}

[1] J. M. Aarts, The structure of orbits in dynamical systems, Fund. Math., 129 (1988), 39-58.

[2] J. M. Aarts and M. Martens, Flows on one-dimensional spaces, Fund. Math., 131 (1988), 53-67.

[3] J. M. Aarts, Orientation of orbits in flows, Papers on General Topology and Related Category Theory and Topological Algebra, Annals of the New York Academy of Sciences, 552 (1989), 1-7.

[4] J. M. Aarts and L. G. Oversteegen, The product structure of homogeneous spaces, Indag. Math., 1 (1990), 1-5.

[5] F. D. Ancel, An alternative proof and applications of a theorem of E. G. Effros, Michigan Math. J., 34 (1987), 39-55.

[6] R. D. Anderson, $A$ characterization of the universal curve and a proof of its homogeneity, Ann. of Math., 67 (1958), 313-324.

[7] R. H. Bing, A homogeneous indecomposable plane continuum, Duke Math. J., 15 (1948), 729-742.

[8] _ Embedding circle like continua in the plane, Canad. J. Math., 14 (1962), 113-126.

[9] E. G. Effros, Transformation groups and $C^{*}$-algebras, Ann. of Math., 81 (1965), 38-55.

[10] R. Engelking, Dimension Theory, Warszawa 1978.

[11] C. L. Hagopian, A characterization of solenoids, Pacific J. Math., 68 (1977), 425-435.

[12] M. W. Mislove and J. T. Rogers, Local product structures on homogeneous continua, preprint. 
[13] E. E. Moise, An indecomposable plane continuum which is homeomorphic to each of its nondegenerate subcontinua, Trans. Amer. Math. Soc., 63 (1948), 581-594.

[14] S. B. Nadler, Hyperspaces of Sets, New York (N. Y.) 1978.

[15] V. V. Nemytskii and V. V. Stepanov, Qualitative Theory of Differential Equations, Princeton (N. J.) 1960.

[16] E. S. Thomas, One-dimensional minimal sets, Topology, 12 (1973), 233-242.

[17] H. Whitney, Regular families of curves, Ann. of Math., 34 (1933), 244-270.

Received December 15, 1988 and in revised form September 22, 1989. Supported in part by NSF-DMS 860-2400 and NSF RII 8610669.

Technical University Delft

Delft, The Netherlands

California State University

SACRAMENTO, CA 95819

AND

University of Alabama at Birmingham

BIRMINGHAM, AL 35294 


\section{PACIFIC JOURNAL OF MATHEMATICS EDITORS}

\author{
V. S. VARADARAJAN \\ (Managing Editor) \\ -University of California \\ Los Angeles, CA 90024-1555-05 \\ Herbert Clemens \\ University of Utah \\ Salt Lake City, UT 84112 \\ THOMAS ENRIGHT \\ University of California, San Diego \\ La Jolla, CA 92093
}

R. FINN

Stanford University

Stanford, CA 94305

Hermann FlaschKa

University of Arizona

Tucson, AZ 85721

VAUGHAN F. R. JONES

University of California

Berkeley, CA 94720

SteVen KerCKhoff

Stanford University

Stanford, CA 94305
C. C. MOORE

University of California

Berkeley, CA 94720

Martin ScharlemanN

University of California

Santa Barbara, CA 93106

\section{HAROLD STARK}

University of California, San Diego

La Jolla, CA 92093

\section{ASSOCIATE EDITORS}

\begin{tabular}{|c|c|c|c|c|}
\hline R. Arens & $\begin{array}{l}\text { E. F. BECKENBACH } \\
(1906-1982)\end{array}$ & Neumann & $\begin{array}{l}\text { F. WolF } \\
(1904-1989)\end{array}$ & K. Yoshida \\
\hline \multicolumn{5}{|c|}{ SUPPORTING INSTITUTIONS } \\
\hline \multicolumn{2}{|c|}{ UNIVERSITY OF ARIZONA } & \multicolumn{3}{|c|}{ UNIVERSITY OF OREGON } \\
\hline \multirow{2}{*}{\multicolumn{2}{|c|}{$\begin{array}{l}\text { UNIVERSITY OF BRITISH COLUMBIA } \\
\text { CALIFORNIA INSTITUTE OF TECHNOLOGY }\end{array}$}} & \multicolumn{3}{|c|}{ UNIVERSITY OF SOUTHERN CALIFORNIA } \\
\hline & & \multicolumn{3}{|c|}{ STANFORD UNIVERSITY } \\
\hline CALIFORNIA INSTITUTE OF TECHNOLOGY & UNIVERSITY OF CALIFORNIA & \multicolumn{3}{|c|}{ UNIVERSITY OF HAWAII } \\
\hline \multicolumn{2}{|c|}{ MONTANA STATE UNIVERSITY } & \multicolumn{3}{|c|}{ UNIVERSITY OF TOKYO } \\
\hline \multicolumn{2}{|c|}{ UNIVERSITY OF NEVADA, RENO } & \multicolumn{3}{|c|}{ UNIVERSITY OF UTAH } \\
\hline \multicolumn{2}{|c|}{ NEW MEXICO STATE UNIVERSITY } & \multicolumn{3}{|c|}{ WASHINGTON STATE UNIVERSITY } \\
\hline OREGON STA & UNIVERSITY & UNIVERS & OF WASHING & \\
\hline
\end{tabular}




\section{Pacific Journal of Mathematics}

Vol. 150, No. $1 \quad$ September, 1991

Jan Aarts, Charles Lemuel Hagopian and Lex Gerard Oversteegen, The orientability of matchbox manifolds $\ldots \ldots \ldots \ldots \ldots \ldots \ldots \ldots \ldots \ldots \ldots \ldots$

Roger W. Barnard, Carl Hanson Fitzgerald and Sheng Gong, The growth and $1 / 4$-theorems for starlike mappings in $\mathbf{C}^{n} \ldots \ldots \ldots \ldots \ldots \ldots \ldots$

Allan Berele, Trace rings for verbally prime algebras $\ldots \ldots \ldots \ldots \ldots \ldots \ldots 23$

Quo-Shin Chi, Curvature characterization and classification of rank-one

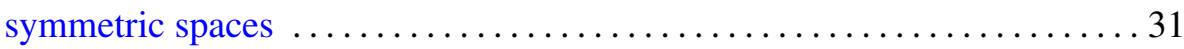

Harold Gerard Donnelly and Jeffrey Marc Lee, Domains in Riemannian

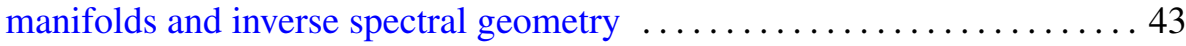

Clifford John Earle, Jr. and Patricia Lilaine Sipe, Families of Riemann

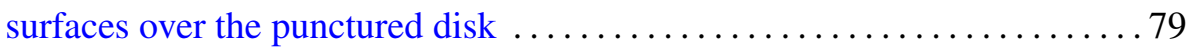

Mark P. Hughes, Dihedral group actions on homotopy complex projective

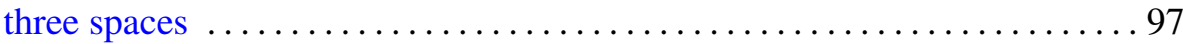

Robert F. Lax and Carl Widland, Gap sequences at a singularity ........111

Takahiko Nakazi, Bounded Hankel forms with weighted norms and lifting

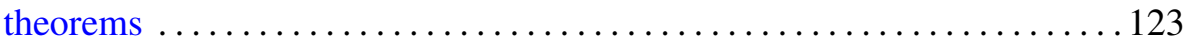

Douglas Murray Pickrell, Mackey analysis of infinite classical motion groups

Martha Rzedowski-Calderón and Gabriel Daniel Villa-Salvador,

Automorphisms of congruence function fields 167

Peter N-S Wong, Equivariant Nielsen fixed point theory for $G$-maps 